\title{
Oxidative toxicity in diabetes and Alzheimer's disease: mechanisms behind ROS/ RNS generation
}

\author{
Waqar Ahmad ${ }^{1 *}$ (D), Bushra ljaz², Khadija Shabbiri', Fayyaz Ahmed² and Sidra Rehman³
}

\begin{abstract}
Reactive oxidative species (ROS) toxicity remains an undisputed cause and link between Alzheimer's disease (AD) and Type-2 Diabetes Mellitus (T2DM). Patients with both AD and T2DM have damaged, oxidized DNA, RNA, protein and lipid products that can be used as possible disease progression markers. Although the oxidative stress has been anticipated as a main cause in promoting both AD and T2DM, multiple pathways could be involved in ROS production. The focus of this review is to summarize the mechanisms involved in ROS production and their possible association with $\mathrm{AD}$ and T2DM pathogenesis and progression. We have also highlighted the role of current treatments that can be linked with reduced oxidative stress and damage in AD and T2DM.
\end{abstract}

Keywords: Alzheimer's disease, Type-2 diabetes mellitus, Oxidative stress, ROS production, Antioxidant treatments, Anti-diabetic drugs

\section{Background}

A set of chemical processes through which living bodies sustain their lives called as metabolism. This includes digestion of food, transport into the body cells and excretion of waste materials through well-conserved intermediary metabolism. The metabolic pathways are the bio-chemical processes involving DNA replication, transcription and translation by enzyme catalysed reaction through which food or other chemicals from the body transformed into different chemicals and produce energy for various life functions [1]. In the living organism's body cells and tissues are always gone through the assembly, and disassembly processes in a regular manner involving several metabolic pathways. Disturbs in metabolic process by any external or internal factors may result in metabolic disorder followed by many types of life-threatening diseases. The understanding of the cellular and molecular mechanism for incurable diseases like Alzheimer's disease (AD) and Type-2 Diabetes Mellitus (T2DM) has been progressing rapidly, which also enhances the therapeutic approaches [2].

\footnotetext{
*Correspondence: waqarchemist@hotmail.com;

waqar.ahmad@uqconnect.edu.au

${ }^{1}$ School of Biological Sciences, University of Queensland, Brisbane 4072, Australia

Full list of author information is available at the end of the article
}

It has been noteworthy that the advancement in diagnostic and therapeutic approaches improved the disease management. However, pathophysiology of many diseases is still under way. $\mathrm{AD}$ and $\mathrm{T} 2 \mathrm{DM}$, the two-utmost communal overwhelming diseases caused by neurological and insulin function disorder, have become a major public health concern worldwide [3, 4], and needed to be address effectively. A large-scale clinico-epidemiological data indicates that both T2DM and AD are most common age-associated diseases around the globe. People with T2DM are prone to risk of $\mathrm{AD}$. The first strong evidence regarding the correlation between $\mathrm{AD}$ and $\mathrm{T} 2 \mathrm{DM}$ was reported in Rotterdam cohort study [3-5]. A number of clinical, epidemiological, biological, molecular and genetic data supports a pathophysiological link between T2DM and AD, including obesity, impaired glucose, cholesterol metabolism, and hypertension [6-8]. Presence of these symptoms altogether known as metabolic syndrome (MetS) and could signify a pathological connection between impaired metabolism and several neurological disorders $[9,10]$. Uncontrolled increased blood glucose is a major cause of T2DM, which is associated with injury of insulin-producing pancreatic $\beta$ cells or by insulin sensitivity in adipose or muscle tissues $[11,12]$. Both T2DM and AD induce disease severity based on same path-physiological mechanisms, including 
mitochondrial damage and formation of advanced glycation products (AGEs). Both mitochondrial damage and AGEs are influenced by induced oxidative stress, which not only impair mtDNA and RNA but also affect protein and lipids $[13,14]$. Several studies found induced levels of DNA, RNA, protein and lipid oxidative products in T2DM and $\mathrm{AD}$ like 8-hydroxyguanosine, 8-hydroxydeoxyguanosine, protein carbonyls and nitrotyrosine; and lipid peroxidation markers, for example, 4-hydroxynonenal, F2-isoprostanes, and malondialdehyde [15-21].

Oxidative stress has been proposed to play a significant role in T2DM and AD progression. The present review highlights the complex mechanism involved in the production of reactive oxygen species (ROS), induced oxidative stress, and their impact on T2DM and AD progression. Moreover, we also highlight the possible treatments to cope with the bad effects of oxidative stress in T2DM and AD.

\section{ROS production and oxidative damage}

ROS in living organisms was first described in 1954 [22, 23]. In 1969, theory of oxygen toxicity was expressed in aerobic organisms after the discovery of superoxide dismutase (SOD) by McCord and Fridovich. ROS production can be associated with age-related diseases, their developmental processes and cell singling pathways $[24,25]$. Oxidative radicals have very short lifespan and react rapidly with other molecules [26]. Presence of transition metals, especially Fe and $\mathrm{Cu}$ can help to clarify and explain oxidative damage to living cells [27]. Important oxidants in the living organism includes ROS, reactive nitrogen species (RNS) and sulphurcentred radicals. Although not all of them are radicals but in many cases, these non-radicals can produce radical species by reacting cellular compounds and damaging them by oxidation [28]. The ROS can be classified into two groups; radicals and non-radicals. The radicals contain superoxide $\left(\mathrm{O}_{2}^{-}\right)$, alkoxyl ( $\left.\mathrm{RO}\right)$, peroxyl (ROO), hydroxyl $\left(\mathrm{OH}^{-}\right)$, hydroperoxyl $\left(\mathrm{HO}_{2}\right)$ and nitric oxide (NO.). The non-radicals include hydrogen peroxide $\left(\mathrm{H}_{2} \mathrm{O}_{2}\right)$, organic peroxides $(\mathrm{ROOH})$, aldehydes (HCOR), hydrochlorous acid (HOCL), peroxynitrite $\left(\mathrm{ONOOH} / \mathrm{ONOO}^{-}\right)$, ozone $\left(\mathrm{O}_{3}\right)$ and singlet oxygen $\left({ }^{1} \mathrm{O}_{2}\right)[29,30]$.

ROS and RNS can be generated through exogenous and endogenous sources [28]. Exogenous sources may include UV radiations (direct oxidation of cellular components) [31, 32], ultrasound, drugs (like narcotics, anaesthetizes, adreamicine, nitroglycerine and belomycinem) [33], food (containing oxidants such as transition metals, aldehydes, fatty acids and peroxides) [34], $\gamma$ - radiations [35], pollutants, xenobiotics and toxic chemicals (alcohol, phosphine, mustard gas) [36, 37]. The endogenous sources may include neutrophils, cytokines and other components of white blood cells $[38,39]$, direct ROS producing enzymes such as NO synthase, indirect ROS producing enzymes such as the xanthin oxidase, mitochondrial, metals and side effects of various diseases [40, 41]. These molecules ultimately target the macromolecules like proteins, lipids and nucleotides that result in genome instability and impaired organ functions [30-34]. These molecules are critical for neuronal and pancreatic beta cell stability and functions [42-44]. ROS readily attacks and generates a variety of variety DNA lesions. These lesions could result in DNA base transversions (e.g. G:C to T:A) [35-37]. More than 200 clinical disorders have been associated with early initiation of ROS. These disorders may include T2DM, AD, cardiovascular damage, inflammation, intestinal tract disease, eye diseases, brain degenerative impairments, aging, hemochromatosis, thalassemia, and Wilson disease $[45,46]$.

In living organisms, Oxidants and antioxidants play a significant role in regulating free radical balance within the body produced during active metabolism. A disturbed endogenous antioxidant system favors shift towards more pro-oxidants production called as "oxidative stress." If it shifts towards more production of antioxidants or reducing power termed as "reductive stress" [25, 30, 47-49]. As induced oxidative stress impairs natural defense by unbalancing the oxidants and de-oxidant ratio, balancing oxidative stress is an emerging therapy in various diseases. Figure 1 explains the detailed mechanism involved in the ROS generation in mitochondria.

\section{ROS as cellular defence}

ROS generally maintains the normal physiological functions and cellular defense of the body. Many living organisms survive below a specific homeostatic set point [24]. Although ROS production is beneficial for cellular mechanism, their excessive quantities are always toxic and lead to oxidative damage of many biological functions $[25,50]$. To reduce its toxicity, mammalian cells have evolved defense mechanisms, including different DNA base excisions and strand repair enzymes [51, 52]. In this way, living organisms have not only adapted themselves to develop self-protective mechanisms for ROS but also able to use it constructively $[24,53]$. Intracellular low level of ROS may act as signaling molecules in many physiological processes, including redox homeostasis and cellular signal transduction [54]. The divergent effects of ROS on many cellular processes suggest that ROS is not merely detrimental by-products, but also generated purposefully to mediate a variety of signaling pathways.

\section{Oxidative stress in T2DM}

$\mathrm{DM}$ is a metabolic disorder categorized into two main groups: Type I (Insulin dependent) that is due to immune-mediated beta-cells destruction and lead to insulin deficiency, and Type 2 (Non-Insulin dependent) that is due to insulin secretion defects and resulted in insulin resistance [55]. Prolonged period of high blood-glucose 
levels generally linked to both macro and micro vascular complications like CVDs, strokes, peripheral vascular diseases, neuropathy, retinopathy and nephropathy [56-58]. In addition to elevated blood-glucose levels, other factors include high-cholesterol level (hyperlipidemia) and oxidative stress leading to high risk of complications [59]. According to epidemiological studies, diabetic mortalities can be explained by an increase in vascular diseases that could be a cause of oxidative damage [60]. Current research reported that apo-lipoprotein component of LDL instead of lipid alone could be a cause of oxidative damage in DM [60].

Production of free radicals and their high levels in diabetic patients could be non-enzymatic (i.e. glycated proteins, glucose oxidation and increased lipid peroxidation) or enzymatic (over/under-expressed levels of enzymes like catalase (CAT), superoxide dismutase (SOD) and glutathione peroxidise (GSH-Px)). These abnormalities may lead to damage of enzymes, cellular machinery and increased insulin resistance due to oxidative stress [61, 62]. Recent studies have provided a clear evidence that the main source of ROS/ RNS production in T2DM is mitochondria [63-65]. Abnormal mitochondrial functions and excessive ROS/RNS production play a primary role in onset T2DM and its complications. These studies also support the possibility for mitochondrial-targeted antioxidant's therapy of T2DM complications [66].

During cellular metabolism, insulin reacts with it receptors that lead to activation of Akt and translocation of GLUT4 to cell membrane. Impaired oxidative phosphorylation, reduced NADH oxidoreductase and citrate synthase activities resulted in insulin resistance $[6,67]$. This insulin resistance could be the result from either impaired fatty acid acetyl-CoA oxidation or from subsequent accumulation of intracellular lipid and diacylglycerol with consequent activation of protein kinase $C$ and ROS production. This impaired fatty acid oxidation resulted in activation of serine kinases followed by phosphorylation of insulin receptor substrates and interfering insulin signal transduction [68].

Multiple studies have observed the presence of oxidative markers like F2-isoprostane and nitrotyrosine in urine, plasma and tissue levels of diabetic patients $[69,70]$. ROS and NOS production in DM can be promoted by both enzymatic and non-enzymatic sources. Main enzymatic sources may be endothelial and vascular smooth muscle cells, NADPH oxidase, xanthine oxidase, cyclooxygenase and uncoupled NOS whereas, non-enzymatic sources include mitochondrial respiratory chain, AGES, glucose autoxidation process and activated polyol pathway [71].

ROS production has become a fundamental part in the T2DM pathogenesis and severity [72]. During the normal glucose oxidation process, the final product is NADH and pyruvate. NADH can reduce pyruvate to lactate or donates its reducing equivalents to electron transport chain. On the other hand, in mitochondrial pyruvate enters into Krebs's cycle, get oxidised and produce $\mathrm{CO}_{2}$, $\mathrm{H}_{2} \mathrm{O}, \mathrm{NADH}$ and $\mathrm{FADH}_{2}$ [73]. In glucose autoxidation, glucose forms radical and converted to reactive ketoaldehydes and superoxide, consequently, produced hydroxyl radical in presence of transition metals via $\mathrm{H}_{2} \mathrm{O}_{2}[74,75]$. Superoxide can also form peroxynitrite radicals by reacting with nitric oxide [76, 77]. Hyperglycemia induced superoxide formation in the mitochondrial electron transport chain by driving the inner mitochondrial membrane potential upward through the generation of excessive electron donors in the Krebs's cycle [78]. This situation resulted in hyperpolarization of mitochondrial membrane potential and increase in ATP/ADP ratio followed by an inhibition of complex-III and electron accumulation at coenzyme Q. Consequently; this situation accelerates free radical formation by partial reduction of $\mathrm{O}_{2}$ and reduces ATP synthesis $[79,80]$.

Superoxide presence decreases glyceraldehyde-3phosphate dehydrogenase (GAPDH) activity by $66 \%$ and resulted in PARP activation and $\mathrm{NAD}^{+}$depletion [81]. In hyperglycemia, glucose conversion to the polyalcohol sorbitol and fructose via the polyol pathway reduces NAD ${ }^{+}$to NADH. Sorbitol oxidation through $\mathrm{NAD}^{+}$escort to increased cytosolic NADH: $\mathrm{NAD}^{+}$ratio and inhibit the GAPDH activity, and consequently, increased production of triose phosphate [80]. Increased triose phosphate induced formation of methylglyoxal and diacylglycerol (DAG), PKC and PARP activation [82, 83]. Hyperglycemia also increases hexosamine pathway flux because of increased bio-availability of nutrients and enhances fructose-6-phosphate levels by inhibiting GAPDH by ROS $[84,85]$. The outcome of the hexosamine pathway is UDP-N-acetyl glucosamine that triggers many transcription factors and pathways, and lead to microvascular complications of T2DM [86, 87].

Overproduction of superoxidase radicals is countered by superoxide dismutase's (SODs) and by uncoupling proteins (UCPs). In hyperglycemia, over expression of UCPs reduce mitochondrial hyperpolarization and ROS formation, and block the glucose induced cell death. Superoxide radical generation was enhanced in patients with diabetic endothelial cells that promote oxidative stress toxicity $[88,89]$. A study by Nishikawa et al. observed the excessive generation of pyruvate via accelerated glycolysis and production of superoxides radicals at the Complex-II level under hyperglycemia [79, 90]. Although glucose is least reactive reducing sugar, it may lead to Amadori product through Schiff base formation by reacting free amino acids. These Aamdori products accumulate on proteins and start the production of AGEs [79, 91] that in turn increase ROS production through binding to RAGE (receptors of AGEs) and 


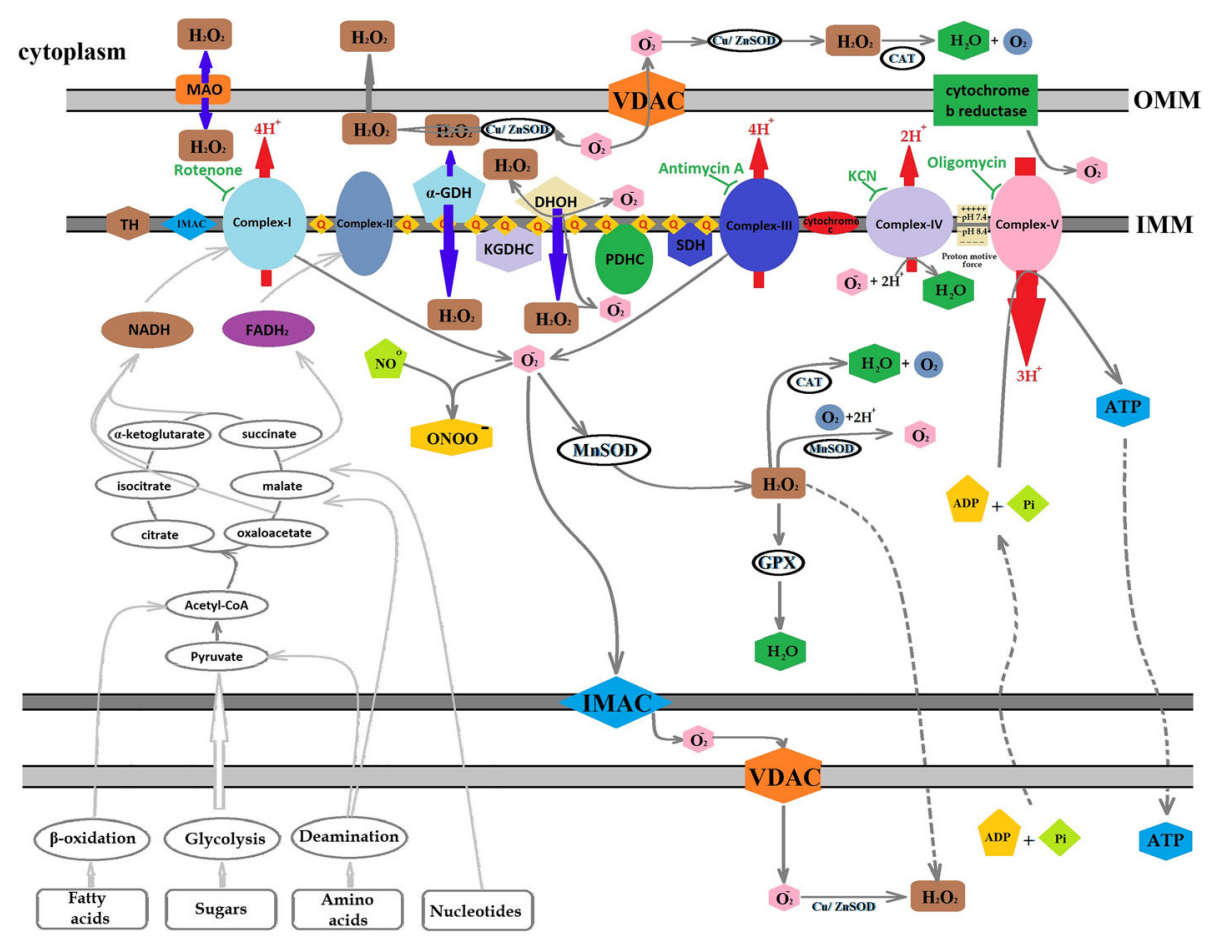

Fig. 1 ROS production in mitochondria. Mitochondria is the primary source for ROS production. There are nine different types of enzymes that have the capacity to generate ROS. Among them, some are present on outer mitochondrial membrane (OMM) i.e. Cytochrome b5 reductase and monoamine oxidases (MAO) and while other found in inner membrane, i.e. dihydroorotate dehydrogenase ( $\mathrm{DHOH})$, dehydrogenase of a-glycerophosphate (a-GDH), succinate dehydrogenase (SDH), aconitase, a-ketoglutarate dehydrogenase complex (KGDHC), Complex-I and Complex-III. MAO, DHOH and a-GDH produces $\mathrm{H}_{2} \mathrm{O}_{2}$ via direct or indirect biochemical reactions, while cytochrome b5, Complex-I and complex-III produce superoxides. Complex-I produced superoxides in presence of $\mathrm{NADH}$ and require tightly bounded ubiquinone. Rotenone can block electron transport by inhibiting ubiquinone and produce ROS, and requires a high degree of redox reduction on the rotenone binding site. The second process involved in ROS production from Complex-I has been known as 'reverse electron transfer (RET)'. In RET, electrons are transferred against the flow of redox potentials of electron carriers (i.e. from reduced co-enzyme Q to $\mathrm{NAD}^{+}$not to oxygen). Complex-III can produce a lot of superoxides during Q-cycle (a multifarious reaction system involved oxidation of coenzyme $\mathrm{Q}$ while, cytochrome c acts as electron carrier/acceptor) that rapidly generate $\mathrm{H}_{2} \mathrm{O}_{2}$ by dismutation. Antimycin can inhibit the quinone reducing site and lead to accumulation of unstable semiquinone and stimulate superoxide production. In the same way KCN and oligomycin can inhibit electron transfer in complex-IV and $V$ respectively, leading to ROS production. SDH is thought to produce ROS via its FAD, while aconitase generate hydroxyl radical by releasing $\mathrm{Fe}^{2+}$. PDHC and KGDHC can produce both superoxides as well as hydrogen peroxide. After generation, superoxide can react with many available molecules or free radicals to form different types of free radicals who can accelerate the cellular damage. To cop superoxide, manganese superoxide dismutase (MnSOD) can convert superoxide to hydrogen peroxide that can be additional converted to water and oxygen by the action of several enzymes like catalase (CAT) or glutathione peroxidase (GPX). For further details, see the text

resulted in the NF-kB induction and NADPH oxidase formation [92, 93]. NADPH oxidase is major source of $\mathrm{O}_{2}^{-}$. Levels of $\mathrm{NADPH}$ and $\mathrm{O}_{2}^{-}$were increased in vascular specimens in diabetic patients [71, 94] and [95]. Binding of AGEs to their receptor RAGE enhanced cytokines and adhesion molecule's production $[96,97]$. This binding also has an abnormal effect on matrix metalloproteinases (MMPs) and transforming growth factor (TGF) $[98,99]$. Hyperglycemia also promotes ROS generation by lipid peroxidation of low-density lipoprotein (LDL) $[100,101]$. Peroxyl radicals produce hydroperoxides by removing one hydrogen from lipids and propagate further [76]. ROS production also induces cellular stresssensitive pathways like NF-kB, JNK/ SAPK, P38 MAPK that leads to cellular damage, and late complications in T2DM [102]. Figure 2 summarizes the mechanism involved in progression of T2DM under high oxidative stress conditions.

\section{Oxidative stress in AD}

Clinically $A D$ is characterized by sinister onset, slowly progressive and sporadic disorder, with episodic memory; instrumental signs include aphasia, apraxia, and agnosia, together with general cognitive symptoms, such as impaired judgment, decision-making, and orientation [103]. There are two opinions about the onset of aging. One view is that, it is genetically programmed developmental processes, like the cell senescence, the neuroendocrine and immunological changes. Another opinion presents that, it is caused by accumulation of somatic mutations and oxidative stress randomly at any time [104]. The crucial events occur during aging progression 


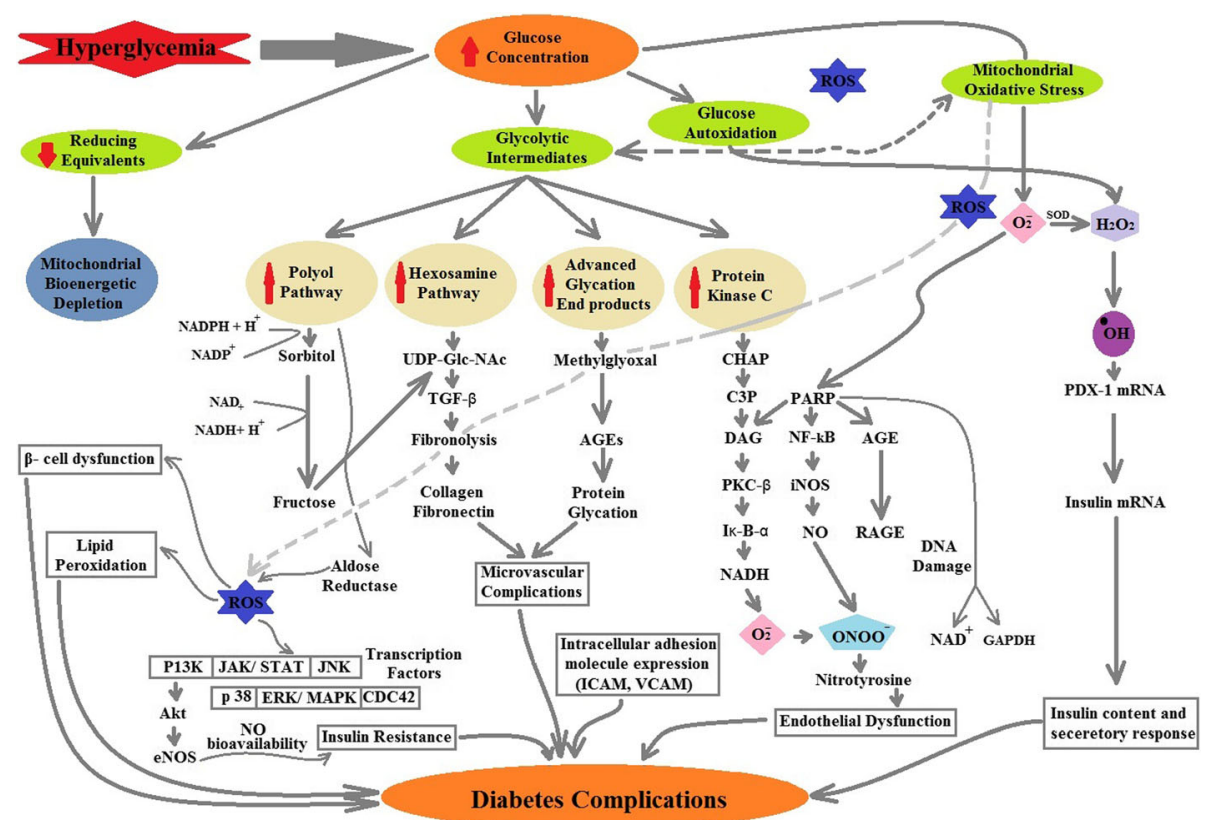

Fig. 2 Oxidative stress production and damage in T2DM. Hyperglycemia is considered as major contributor in ROS production and associated- damage in T2DM. Induced glucose concentrations may have led to glucose autoxidation, impaired mitochondrial bioenergetics and over production of ROS. Induced oxidative stress in T2DM can impair a couple of transcription factors and pathways like P13K, JAK/STAT, JNK, p-38, ERK/MAPK and CDC42 that resulted in insulin resistance. The other glycolytic intermediates can have led to microvascular complications and endothelial dysfunctions and prone to several diabetic complications

or their onsets are telomere erosion, oxidative stress and cell senescence. Aged cell phenotype showed futile ROS regulation on mitochondrial super-complexes that causes ROS signalling changes [105]. The neuronal cells are highly sensitive and susceptible to oxidative stress as a result of its high intake of oxygen, lipid content and scantiness of antioxidant enzymes as compared to normal other body tissues [106]. It has been shown that with the passage of time and advance age, ratios of ROS production and antioxidant activities (superoxide dismutase and catalase or glutathione peroxidase enzymes) are disturbed and oxidative damage of macromolecules and their product's build-up in the brain [107-109].

One of the hallmarks of $\mathrm{AD}$ is the accumulation of amyloid beta $(A \beta)$ peptide mostly in mitochondria and it has been shown that $\mathrm{A} \beta$ peptide itself can generate ROS in the presence of metal ions such as $\mathrm{Fe}^{+}$and $\mathrm{Cu}^{+}$ [110]. In mouse models and autopsy analysis of AD patients, mitochondrial dysfunction leads to increased ROS or increased ROS production lead to mitochondrial dysfunction, which in turn enhances $A \beta$ peptide aggregation. Importantly, these elevated markers for oxidative stress precede $A \beta$ deposition and neurofibrillary tangles, suggesting that oxidative stress is an early event involved in $\mathrm{AD}$ pathogenesis. Abnormal production of proteins and mtDNA mutation may be due to defective or deficient base excision repair (BER) enzymes and its associated pathways [111-114].
Several hypotheses described oxidative stress as a main culprit in AD pathophysiology $[115,116]$. The nervous system is rich source of unsaturated fatty acids and iron. Both these high lipid and iron contents become the targets for oxidative damage in nervous system. In $\mathrm{AD}$ pathology, decline in synaptic activities, defects and low energy metabolism with comparatively increased amount of $\mathrm{ROS}$, reduced antioxidants enzymes levels like $\mathrm{Cu} /$ $\mathrm{Zn}$-SOD, glutathione (GSH) and catalase in frontal and temporal cortex, and presence of $\mathrm{A} \beta$ and NFTs together lead to mitochondrial dysfunctions and neuronal cell death. There are many mechanisms responsible for oxidative stress, like sugar modifications, peroxidation of lipids, oxidation of protein DNA/RNA and production of free radicals by $A \beta$ itself. These molecules are critical for neuronal stability and functions [42-44]. In AD patients, the free-radical production is intimately associated with unique sources of $\mathrm{AD}$ pathology. The $\mathrm{A} \beta$ (formed by proteolysis of a transmembrane glycoprotein $A \beta$ precursor protein ( $\beta-A P P))$ component of senile plaques is main source of free radical production once it formed outside the neurons via metal-catalysed oxidation of APP [117, 118]. Metals, especially iron plays a significant role in free radical production in AD. Increased iron contents have been found in $\mathrm{A} \beta$ and NFTs deposits that catalyses hydrogen peroxide $\left(\mathrm{H}_{2} \mathrm{O}_{2}\right)$ and form hydroxyl radicals by Fenton reaction. $\mathrm{A} \beta$ is also able to boost up the metal ions (such as iron, aluminium 
and copper) capacity to generate free radicals. $A \beta$ has been shown to produce $\left(\mathrm{H}_{2} \mathrm{O}_{2}\right)$ and releasing thiobabituric acid reactive substances (TBARS) mainly associated with hydroxyl radicals $(\mathrm{OH})$ via metal ion reduction. $A \beta$ also induce neurodegeneration by targeting microglial NADPH oxidase however, mechanism behind this destruction is poorly understood [119].

AGEs that are present in the senile plaques also produce free radicals by chemical oxidation and degradation, by binding to their receptors (RAGE) or interacting with microglia that surrounds the senile plaques. It results in respiratory blast and production of superoxides and $\mathrm{NO}$ $[120,121]$. The membranes from the brain are composed of proteins and phospholipids. Presence of aluminium in NFTs stimulates iron-induced lipid peroxidation of oxidisable polyunsaturated fatty acids (PUFAs) that contain weak double bond hydrogen atoms. These PUFAs (like arachidonic acid, docosahexaenoic acid) resulted in multiple aldehydes like acrolein and 4-hydroxy-2-nonenal (HNE). HNE accumulation was shown in NFTs may cause tau phosphorylation, damage or kill primary hippocampus neurons, gene induction, crosslinking of cytoskeletal proteins, cytotoxicity and inhibition of cyclins D1 and D2. HNE also disrupts the binding of histones to DNA and increases chances of DNA oxidation in AD brain [122]. F2-isoprostanes a lipid reliable peroxidation marker is also produced from non-enzymatic peroxidation of arachidonic acid [123].

The oxidation of amino acids like lysine, arginine, proline and histidine via peroxynitrite generates protein carbonyls and nitrile that were increased in $\operatorname{AD}[124,125]$. Increased levels of protein carbonyls may decrease ATP availability in synaptic terminals and disrupt the cytoskeletal protein assembly [125]. The protein oxidation via nitric oxide produce ONOO radical and nitrotyrosine that are important non-invasive marker for protein oxidation in AD $[125,126]$. The other protein's oxidation such as ubiquitin, methionine and cysteine is associated with NFTs and the number of tangles has inverse relation with soluble proteins. [127].

The oxidation of DNA and RNA especially mtDNA in $\mathrm{AD}$ results in hydroxylated base's products, DNA-protein crosslinking, strand breakage and impairment of DNA repair system. The levels of $8 \mathrm{OHdG}$ were high in $\mathrm{AD}$ when compared to the age-matched controls [128, 129]. RNA oxidation is a primary target in $\mathrm{AD}$ as RNA is less secure than DNA due to single stranded and specific proteins like histones. The non-coding RNAs are also involved in synapsis, neuronal specification and differentiation, and regulation of dendritic spine development. So their damage due to oxidative stress contributes in development of neurodegenerative diseases specially AD [130, 131]. Nunamara et al., extensively reviewed the RNA oxidation in neurodegenerative diseases and discussed the biological significance and cellular mechanism against RNA oxidation [132].
As mitochondria are concerned with a regulatory role in cells through apoptosis, their dysfunction due to oxidative stress may lead a disruption of cellular functions [133, 134]. Apoptosis activates caspases via proteins like BAD, BOX and results in morphological and biochemical changes leading to cell death whereas anti-apoptotic protein $\mathrm{BCL}-2$ over expression may reduce $A \beta$-induced toxicity in $A D$ via inhibiting $\mathrm{p} 38$, MAPK and NFkB proapoptotic activation [135-137]. A $\beta$ presence also decreased the mitochondrial respiratory chain complexes activity, while the activity of ATP synthase $\alpha$-chain reduced with accumulation of NFTs $[129,138,139]$. Figure 3 highlighted the important pathways involved in damage created by oxidative stress in $\mathrm{AD}$.

The effect of oxidative stress on both T2DM and AD remained to define. Intervention to excessive ROS production through scavenging free radicals and increasing antioxidant defence mechanisms are extensively anticipated as anti-aging therapy and also managing $\mathrm{AD}$ and T2DM. However, positive and conclusive results have not been achieved even with the association of supplementation and pharmacological or natural compounds. It is possible that few antioxidants may become useless or even harmful sooner or later. Supporting evidence has been obtained from the previous research, which indicates the significant role of oxidative stress in the development of neuronal injury in the diabetic brain and the beneficial effects of antioxidants. We must take into account, that research studies also reported on the failure of antioxidant's therapies for T2DM. In contrast, the ongoing large clinical trials will also shed additional light on the clinical merit of antioxidant supplementation [66, 140]. These studies suggest that the clearly linking products i.e. deregulated ROS production and oxidative stress in both disorders may lead to common therapy.

\section{Conclusions}

The multi-factorial and inexorable phenomenon of disease complexity of both T2DM and AD leads to gradual reduction of resistance towards oxidative stress, and metabolic disorders that are the major hallmarks of both illnesses. Genetic studies have improved our understanding of pathways that lead to both disorders that highlighting possible interventional targets. Association between $\mathrm{AD}$ and T2DM suggests that drug givens to $\mathrm{AD}$ patients would be more effective as given to DM [6, 114, 141, 142]. Therefore, targeting T2DM might be more constructive for treating AD. It is also suggested that drugs which used to treat T2DM may affect AD progression ether directly in the brain, provided they pass the blood-brain barrier or indirectly, by modification of systemic blood-glucose concentrations, insulin, inflammatory markers and AGEs. Hence, recent research mostly focuses on treating $\mathrm{AD}$ through anti-diabetic drugs that have a direct effect on the 


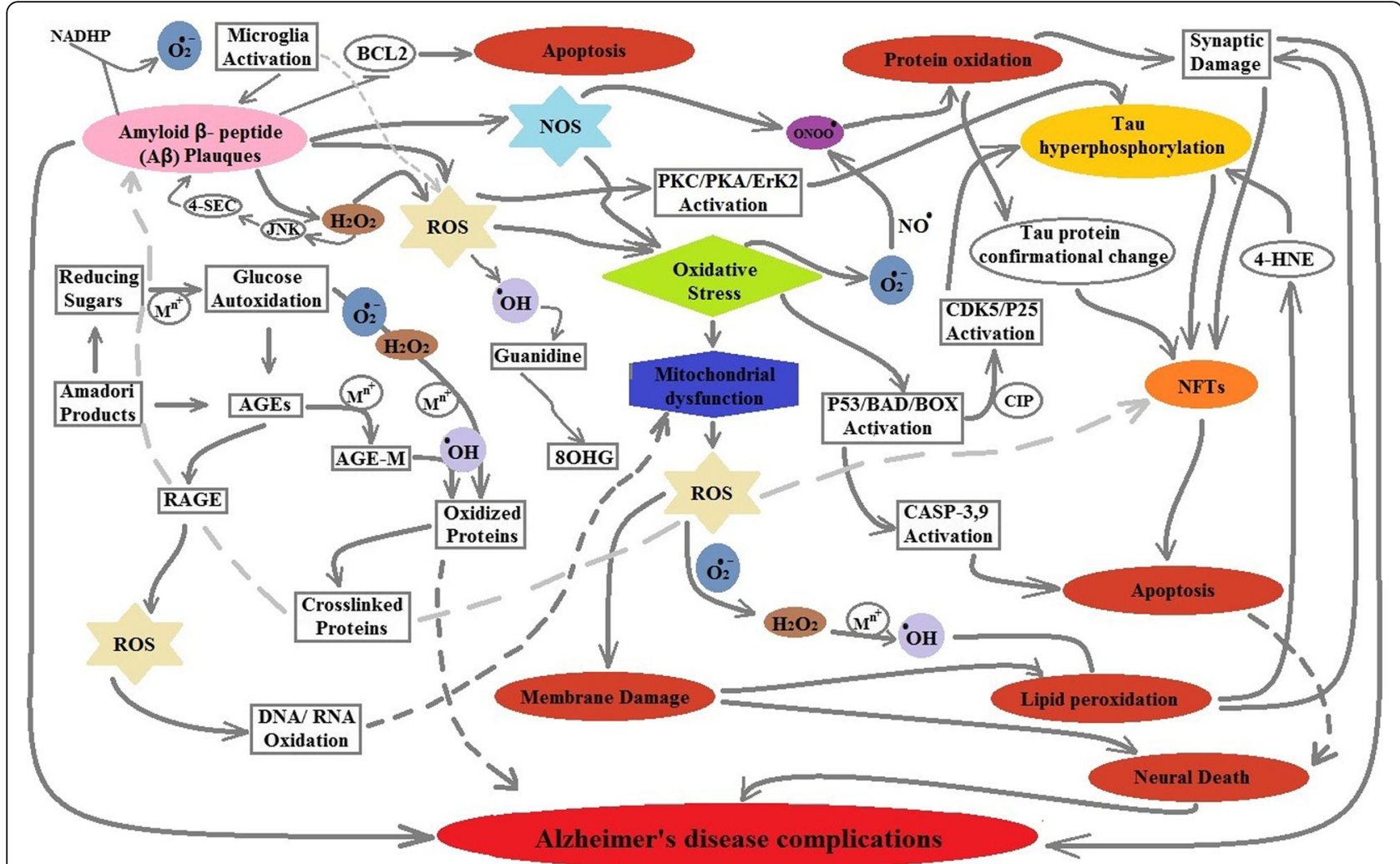

Fig. 3 Production and mechanism of oxidative stress in AD. Brain consumes more oxygen than the whole body, and is a rich source of fatty acids and metals that are more susceptible to oxidative damage in AD. Two main hallmarks of AD i.e. A $\beta$ plaques and hyper-phosphorylated tau neurofibrillary tangles (T-NFTs) are involved in production as well as promotion of oxidative damage. Any abnormal increase in ROS due to presence of A $\beta$ and NFTs promote mitochondrial DNA RNA damage that resulted in mitochondrial dysfunction and membrane damage. Other damages associated with oxidative stress in $A D$ are autoxidation of glucose that resulted in production of AGES and alternatively induce $A \beta$ - toxicity. As oxidative stress, itself induce $A \beta$ and NFTs formation, the result is induced apoptosis, neuronal death and impaired synapsis

brain tissue since brain insulin resistance is often associated with AD [143]. Preclinical and postmortem neuropathological studies have identified significant effect of normal insulin signaling in proper functioning through the brain. These findings have given way for investigating novel therapeutic agents for common $\mathrm{AD}$ and T2DM pathways [6].

Epidemiological research data has substantiated a strong linkage between T2DM and AD whereas the exact mechanism behind this enhanced risk yet to be discovered. Both $\mathrm{AD}$ and T2DM have a high incidence rate at advanced age. Several recent researches reported communal pathological causes between T2DM and AD and therefore, common preventive and therapeutic agents might be effective for both types of disease. The oxidative stress has a transitional part in the $\mathrm{AD}$ development. More research is requisite for explore explosive rate in T2DM in the younger generation. Unfortunately, observations made for T2DM and AD drugs seemed to be working in vertebrate and invertebrate models of T2DM, but appears to fail during clinical trials except intranasal insulin therapy. Considering present review, enzyme inhibition is also answering and promising strategy against both types of disease. However, its role in patho-physiology and therapeutics is still needed to explore fully. In conclusion, shared pathogenesis and curative agents make possible to manage life style pattern and use of new therapeutic agents.

\section{Future perspectives}

A better understanding of oxidative stress production and coping in the AD and T2DM might offer some novel targets for therapy. It is further to point out that whether oxidative stress is the eventual basis of pathogenesis; anti-oxidant therapy gets the reward for ultimate treatment. The strategy should be designed in aims of specifically targeting free radical production and oxidative stress that limit its production and progression in the body but how is it possible? Natural products, which are extensively studied to control different diseases by hindering or suppressing ROS production, might be a good choice. Further work is required for better understanding the role of oxidative stress in $\mathrm{AD}$ and $\mathrm{T} 2 \mathrm{DM}$ progression hence new techniques are compulsory against these 
substances. Poor knowledge of basic mechanisms involved in aging process, which might interfere to prevent or delay age-related pathologies, like T2DM, cardiovascular disorders, neurodegenerative disorders, and cancer. More investigations are clearly needed to clarify the discrepancy in the role of ROS and antioxidant enzymes in aging process and age-related diseases and to understand the precise role of free radicals play in that processes.

\section{Abbreviations}

AD: Alzheimer's disease; AGEs: Advanced glycation end products; A $\beta$ : Betaamyloid; BBB: Blood brain barrier; DAG: Diacylglycerol; GAPDH: Glyceraldehyde-3phosphate dehydrogenase; GSH: Glutathione peroxidise; GSK3: Glycogen synthase kinase3; $\mathrm{H}_{2} \mathrm{O}_{2}$ : hydrogen peroxide; $\mathrm{HNE}$ : 4-hydroxy-2-nonenal; $\mathrm{KGDHC}$ : aKetoglutarate dehydrogenase complex; LDL: Low-density lipoprotein; LMWA: Low molecular weight antioxidants; MAO: Monoamine oxidases; MetS: Metabolic syndrome; MMPs: Matrix metalloproteinases; MPO: Myeloperoxidase; NADH: Nicotinamide dinucleotide; OMM: Outer mitochondrial membrane; RET: Reverse electron transfer; RNS: Reactive nitrogen species; ROS: Reactive oxygen species; SOD: Superoxide dismutase; T2DM: Type-2 diabetes mellitus; TZDs: Thiazolidinediones; a -GDH: a-glycerophosphate

\section{Acknowledgements}

Not Applicable.

\section{Funding}

Not Applicable.

\section{Availability of data and materials}

Not Applicable.

\section{Authors' contributions}

WA, BI, KS, FA, and SR wrote the different parts of the manuscript. WA and KS draw the pictures included in this manuscript. WA and Bl edited the manuscript. All authors read and approved the final manuscript.

\section{Authors information}

Waqar Ahmad is working as Research Officer while Khadija Shabbiri is post-doctoral Research fellow at School of Biological Sciences, University of Queensland, Australia. Bushra ljaz is Assistant professor at CEMB, University of the Punjab, Lahore, Sidra Rehman is Assistant professor at COMSATS; while Fayyaz Ahmed is PhD a student.

\section{Ethics approval and consent to participate}

Not Applicable.

\section{Consent for publication}

The authors declare that this article is original and never been published before and not submitted to any other journal.

\section{Competing interests}

The authors have no competing interests.

\section{Publisher's Note}

Springer Nature remains neutral with regard to jurisdictional claims in published maps and institutional affiliations.

\section{Author details}

${ }^{1}$ School of Biological Sciences, University of Queensland, Brisbane 4072, Australia. ${ }^{2}$ Centre of Excellence in Molecular Biology, University of the Punjab, Thokar Niaz Baig, Lahore 54000, Pakistan. ${ }^{3}$ COMSATS Institute of Information Technology Abbottabad, Abbottabad 22010, Pakistan.
Received: 5 January 2017 Accepted: 5 September 2017

Published online: 19 September 2017

\section{References}

1. Mulukutla BC, et al. Regulation of Glucose Metabolism - A Perspective From Cell Bioprocessing. Trends Biotechnol. 2016;34(8):638-51.

2. McNamara JT, Morgan JL, Zimmer J. A molecular description of cellulose biosynthesis. Annu Rev Biochem. 2015;84:895-921.

3. Berchtold NC, Cotman CW. Evolution in the conceptualization of dementia and Alzheimer's disease: Greco-roman period to the 1960s. Neurobiol Aging. 1998;19(3):173-89.

4. Münch $\mathrm{G}$, et al. Alzheimer's Disease-synergistic effects of glucose deficit, oxidative stress and advanced glycation endproducts. J Neural Transm. 1998;105(4-5):439-61.

5. Hofman A, et al. The Rotterdam Study: 2016 objectives and design update. Eur J Epidemiol. 2015;30(8):661-708. PMID:26386597; PMCID:PMC4579264. doi:10.1007/s10654-015-0082-x.

6. Ahmad W. Overlapped metabolic and therapeutic links between Alzheimer and diabetes. Mol Neurobiol. 2013;47(1):399-424.

7. Mittal K, Katare DP. Shared links between type 2 diabetes mellitus and Alzheimer's disease: A review. Diabetes Metab Syndr. 2016;10(2 Suppl 1):S144-9.

8. Rosales-Corral $\mathrm{S}$, et al. Diabetes and Alzheimer disease, two overlapping pathologies with the same background: oxidative stress. Oxidative Med Cell Longev. 2015;2015:985845.

9. Biessels G-J, et al. Cerebral function in diabetes mellitus. Diabetologia. 1994; 37(7):643-50.

10. Takeuchi M, Yamagishi S-i. Possible involvement of advanced glycation endproducts (AGEs) in the pathogenesis of Alzheimer's disease. Curr Pharm Des. 2008;14(10):973-8

11. Biessels $G$, Kappelle L. Increased risk of Alzheimer's disease in type II diabetes: insulin resistance of the brain or insulin-induced amyloid pathology? Biochem Soc Trans. 2005;33(5):1041-4.

12. Butterfield DA, Di Domenico F, Barone E. Elevated risk of type 2 diabetes for development of Alzheimer disease: a key role for oxidative stress in brain. Biochim Biophys Acta. 2014;1842(9):1693-706.

13. Montecalvo MA, et al. Natural history of colonization with vancomycin-resistant Enterococcus faecium. Infect Control Hosp Epidemiol. 1995;16(12):680-5.

14. Reser JE. Alzheimer's Disease and natural cognitive aging may represent adaptive metabolism reduction programs. Behav Brain Funct. 2009:5(1):1.

15. Wang J, Yang X, Zhang J. Bridges between mitochondrial oxidative stress, ER stress and mTOR signaling in pancreatic beta cells. Cell Signal. 2016;28(8):1099-104.

16. Sadeghi A, et al. The effect of diabetes mellitus on apoptosis in hippocampus: cellular and molecular aspects. Int J Prev Med. 2016;7:57.

17. Zheng $\mathrm{H}$, et al. Protein modifications as manifestations of hyperglycemic Glucotoxicity in diabetes and its complications. Biochem Insights. 2016;9:1-9.

18. Ceriello A, Testa R, Genovese S. Clinical implications of oxidative stress and potential role of natural antioxidants in diabetic vascular complications. Nutr Metab Cardiovasc Dis. 2016;26(4):285-92.

19. Tramutola A, et al. Oxidative stress, protein modification and Alzheimer disease. Brain Res Bull. 2017;133:88-96.

20. Grimm A, Mensah-Nyagan AG, Eckert A. Alzheimer, mitochondria and gender. Neurosci Biobehav Rev. 2016;67:89-101.

21. Cervellati $\mathrm{C}$, et al. Oxidative challenge in Alzheimer's disease: state of knowledge and future needs. J Investig Med. 2016;64(1):21-32.

22. Commoner B, Townsend J, Pake GE. Free radicals in biological materials. Nature. 1954;174(4432):689-91.

23. Newsholme P, et al. Molecular mechanisms of ROS production and oxidative stress in diabetes. Biochem J. 2016:473(24):4527-50.

24. Finkel T, Holbrook NJ. Oxidants, oxidative stress and the biology of ageing. Nature. 2000;408(6809):239-47.

25. Gracy R, et al. Reactive oxygen species: the unavoidable environmental insult? Mutat Res Fundam Mol Mech Mutagen. 1999;428(1):17-22.

26. Bansal AK, Bilaspuri G. Impacts of oxidative stress and antioxidants on semen functions. Vet Med Int. 2010;2011

27. Halliwell B, Gutteridge J. Biologically relevant metal ion-dependent hydroxyl radical generation an update. FEBS Lett. 1992;307(1):108-12.

28. Kohen R, Gati I. Skin low molecular weight antioxidants and their role in aging and in oxidative stress. Toxicology. 2000;148(2):149-57.

29. Phaniendra A, Jestadi DB, Periyasamy L. Free radicals: properties, sources, targets, and their implication in various diseases. Indian J Clin Biochem. 2015;30(1);11-26. 
30. Kohen R, Moor E, Oron M. Measurements of biological reducing power in health and diseases by voltammetric methods. Redox Genome Interaction Health Dis. 2004:13-42.

31. Victorin K. Review of the genotoxicity of nitrogen oxides. Mutat Res. 1994; 317(1):43-55.

32. Koren HS. Associations between criteria air pollutants and asthma. Environ Health Perspect. 1995;103(Suppl 6):235.

33. Chinev $S$, et al. Lipid peroxidation in rat lung induced by neuroleptanalgesia and its components. Eur J Anaesthesiol. 1998;15(6):686-94.

34. Sen C, Packer L, Hänninen O. Biological thiols and redox regulation of cellular signal transduction pathways. Handbook of oxidants and antioxidants in exercise. Amsterdam: Elsevier; 2000. p. 375-402.

35. Shadyro O, Yurkova I, Kisel M. Radiation-induced peroxidation and fragmentation of lipids in a model membrane. Int J Radiat Biol. 2002;78(3):211-7.

36. Elsayed NM, et al. Free radical-mediated lung response to the monofunctional sulfur mustard butyl 2-chloroethyl sulfide after subcutaneous injection. Toxicology. 1992;72(2):153-65.

37. Obata T, et al. Release of dopamine by perfusion with 1-methyl-4phenylpyridinium ion (MPP+) into the striatum is associated with hydroxyl free radical generation. Brain Res. 2001;906(1):170-5.

38. Dhalla NS, Temsah RM, Netticadan T. Role of oxidative stress in cardiovascular diseases. J Hypertens. 2000;18(6):655-73.

39. Forman HJ, Torres M. Reactive oxygen species and cell signaling: respiratory burst in macrophage signaling. Am J Respir Crit Care Med. 2002; 166(supplement_1):S4-8.

40. Fleury $C$, Mignotte $B$, Vayssière J-L. Mitochondrial reactive oxygen species in cell death signaling. Biochimie. 2002;84(2):131-41.

41. Gutteridge JM, Halliwell B. Invited review free radicals in disease processes: a compilation of cause and consequence. Free Radic Res Commun. 1993; 19(3):141-58.

42. Dong J, et al. Metal binding and oxidation of amyloid- $\beta$ within isolated senile plaque cores: Raman microscopic evidence. Biochemistry. 2003;42(10): 2768-73.

43. Hoyer S. Causes and consequences of disturbances of cerebral glucose metabolism in sporadic Alzheimer disease: therapeutic implications. Adv Exp Med Biol. 2004:541:135-52.

44. Butterfield DA, Pocernich CB. The glutamatergic system and Alzheimer's disease. CNS drugs. 2003;17(9):641-52

45. Kaul M, Garden GA, Lipton SA. Pathways to neuronal injury and apoptosis in HIV-associated dementia. Nature. 2001:410(6831):988-94.

46. Bielski $\mathrm{BH}$, et al. Reactivity of $\mathrm{HO} 2 / \mathrm{O}-2$ radicals in aqueous solution. J Phys Chem Ref Data. 1985;14(4):1041-100.

47. Schafer FQ, Buettner GR. Redox environment of the cell as viewed through the redox state of the glutathione disulfide/glutathione couple. Free Radic Biol Med. 2001;30(11):1191-212

48. Kohen R, Nyska A. Oxidation of biological systems: oxidative stress phenomena, antioxidants, redox reactions, and methods for their quantification. Toxicol Pathol. 2002;30(6):620-50.

49. Hrbac J, Kohen R. Biological redox activity: its importance, methods for its quantification and implication for health and disease. Drug Dev Res. 2000; 50(3-4):516-27.

50. Comporti M. Three models of free radical-induced cell injury. Chem Biol Interact. 1989:72(1):1-56.

51. Priora R, et al. Measurement of mixed disulfides including glutathionylated proteins. Methods Enzymol. 2010;473:149-59.

52. Muralidharan $P$, et al. Evidence for redox sensing by a human cardiac calcium channel. Sci Rep. 2016:6

53. Dröge W. Free radicals in the physiological control of cell function. Physiol Rev. 2002;82(1):47-95.

54. Newsholme $P$, et al. Nutrient regulation of insulin secretion and action. J Endocrinol. 2014;221(3):R105-20.

55. Salsali A, Nathan M. A review of types 1 and 2 diabetes mellitus and their treatment with insulin. Am J Ther. 2006;13(4):349-61.

56. Wallace T, Matthews D. Recent advances in the monitoring and management of diabetic ketoacidosis. QJM. 2004;97(12):773-80.

57. Asmat U, Abad K, Ismail K. Diabetes mellitus and oxidative stress-a concise review. Saudi Pharm J. 2016;24(5):547-53.

58. Loghmani E. Diabetes mellitus: type 1 and type 2. Guidelines for adolescent nutrition services; 2005. p. 167-82.

59. Kangralkar V, Patil SD, Bandivadekar R. Oxidative stress and diabetes: a review. Int J Pharm Appl. 2010;1(1):38-45.
60. Pham-Huy LA, He H, Pham-Huy C. Free radicals, antioxidants in disease and health. Int J Biomed Sci. 2008;4(2):89-96.

61. Maritim A, Sanders a, Watkins r J. Diabetes, oxidative stress, and antioxidants: a review. J Biochem Mol Toxicol. 2003;17(1):24-38.

62. Lipinski B. Pathophysiology of oxidative stress in diabetes mellitus. J Diabetes Complicat. 2001;15(4):203-10.

63. Moussa S. Oxidative stress in diabetes mellitus. Romanian J Biophys. 2008; 18(3):225-36.

64. Erejuwa $\mathrm{OO}$. Oxidative stress in diabetes mellitus: is there a role for hypoglycemic drugs and/or antioxidants. Oxidative stress and diseases; 2012. p. 217-46.

65. Ceriello PA. Oxidative stress and diabetes-associated complications. Endocr Pract. 2006;12(Supplement 1):60-2.

66. Calkins MJ, Manczak M, Reddy PH. Mitochondria-targeted antioxidant SS31 prevents amyloid beta-induced mitochondrial abnormalities and synaptic degeneration in Alzheimer's disease. Pharmaceuticals. 2012:5(10):1103-19.

67. Jahangir Z, Ahmad W, Shabbiri K. Alternate Phosphorylation/O-GlcNAc modification on human insulin IRSs: a road towards impaired insulin signaling in Alzheimer and diabetes. Adv Bioinforma. 2014;2014:324753.

68. Sorriento D, et al. Targeting mitochondria as therapeutic strategy for metabolic disorders. ScientificWorldJournal. 2014;2014:604685.

69. Guzik TJ, et al. Vascular superoxide production by NAD (P) H oxidase association with endothelial dysfunction and clinical risk factors. Circ Res. 2000;86(9):e85-90.

70. Ceriello A, et al. Detection of nitrotyrosine in the diabetic plasma: evidence of oxidative stress. Diabetologia. 2001:44(7):834-8.

71. Guzik TJ, et al. Mechanisms of increased vascular superoxide production in human diabetes mellitus role of NAD (P) H oxidase and endothelial nitric oxide synthase. Circulation. 2002;105(14):1656-62.

72. Pazdro R, Burgess JR. The role of vitamin $E$ and oxidative stress in diabetes complications. Mech Ageing Dev. 2010;131(4):276-86.

73. Brownlee M. Internationale Fachliteratur. Nature. 2001;414(6865):813-20.

74. Jiang Z-Y, Woollard A, Wolff SP. Hydrogen peroxide production during experimental protein glycation. FEBS Lett. 1990;268(1):69-71.

75. Wolff SP. Dean R. Glucose autoxidation and protein modification. The potential role of 'autoxidative glycosylation'in diabetes. Biochem J. 1987; 245(1):243-50

76. Halliwell B, Gutteridge JM. [1] Role of free radicals and catalytic metal ions in human disease: an overview. Methods Enzymol. 1990;186:1-85.

77. Hogg N, et al. Inhibition of low-density lipoprotein oxidation by nitric oxide potential role in atherogenesis. FEBS Lett. 1993;334(2):170-4.

78. Korshunov SS, Skulachev VP, Starkov AA. High protonic potential actuates a mechanism of production of reactive oxygen species in mitochondria. FEBS Lett. 1997;416(1):15-8

79. Brownlee M. Biochemistry and molecular cell biology of diabetic complications. Nature. 2001:414(6865):813-20.

80. Nishikawa T, Edelstein D, Brownlee M. The missing link: a single unifying mechanism for diabetic complications. Kidney Int. 2000:58:S26-30.

81. Pacher P, Szabó C. Role of poly (ADP-ribose) polymerase-1 activation in the pathogenesis of diabetic complications: endothelial dysfunction, as a common underlying theme. Antioxid Redox Signal. 2005;7(11-12):1568-80.

82. Williamson JR, et al. Hyperglycemic pseudohypoxia and diabetic complications. Diabetes. 1993;42(6):801-13.

83. Choudhury, M., Involvement of Caspase-7 in photoreceptor and retinal ganglion cell death. 2014.

84. Zhang Z, et al. High glucose inhibits glucose-6-phosphate dehydrogenase via CAMP in aortic endothelial cells. J Biol Chem. 2000;275(51):40042-7.

85. James $L R$, et al. Flux through the Hexosamine pathway is a determinant of nuclear factor KB-dependent promoter activation. Diabetes. 2002;51(4):1146-56.

86. McClain DA, Crook ED. Hexosamines and insulin resistance. Diabetes. 1996; 45(8):1003-9.

87. Goldberg HJ, Whiteside $\mathrm{Cl}$, Fantus IG. The hexosamine pathway regulates the plasminogen activator inhibitor-1 gene promoter and Sp1 transcriptional activation through protein kinase C- $\beta$ I and- $\delta$. J Biol Chem. 2002:277(37):33833-41

88. Du X-L, et al. Hyperglycemia-induced mitochondrial superoxide overproduction activates the hexosamine pathway and induces plasminogen activator inhibitor-1 expression by increasing Sp1 glycosylation. Proc Natl Acad Sci. 2000;97(22):12222-6.

89. Li L-X, et al. Uncoupling protein-2 participates in cellular defense against oxidative stress in clonal $\beta$-cells. Biochem Biophys Res Commun. 2001; 282(1):273-7. 
90. Nishikawa T, et al. Normalizing mitochondrial superoxide production blocks three pathways of hyperglycaemic damage. Nature. 2000;404(6779):787-90.

91. Ulrich P, Cerami A. Protein glycation, diabetes, and aging. Recent Prog Horm Res. 2000:56:1-21.

92. Baynes JW, Thorpe SR. Role of oxidative stress in diabetic complications: a new perspective on an old paradigm. Diabetes. 1999;48(1):1-9.

93. McCarthy A, Etcheverry S, Cortizo A. Effect of advanced glycation endproducts on the secretion of insulin-like growth factor-l and its binding proteins: role in osteoblast development. Acta Diabetol. 2001;38(3):113-22.

94. Ergul A, et al. Vascular dysfunction of venous bypass conduits is mediated by reactive oxygen species in diabetes: role of endothelin-1. J Pharmacol Exp Ther. 2005;313(1):70-7.

95. Amiri F, et al. Angiotensin II activation of the JAK/STAT pathway in mesangial cells is altered by high glucose. Kidney Int. 2002;61(5):1605-16.

96. Wake $\mathrm{H}_{\text {, et }}$ al. Histamine inhibits advanced glycation end products-induced adhesion molecule expression on human monocytes. J Pharmacol Exp Ther. 2009;330(3):826-33.

97. Takahashi HK, et al. Advanced glycation end products subspecies-selectively induce adhesion molecule expression and cytokine production in human peripheral blood mononuclear cells. J Pharmacol Exp Ther. 2009;330(1):89-98.

98. Lohwasser $\mathrm{C}$, et al. The receptor for advanced glycation end products is highly expressed in the skin and upregulated by advanced glycation end products and tumor necrosis factor-alpha. J Investig Dermatol. 2006;126(2):291-9.

99. Hergrueter AH, Nguyen K, Owen CA. Matrix metalloproteinases: all the RAGE in the acute respiratory distress syndrome. Am J Physiol Lung Cell Mol Physiol. 2011;300(4):L512-5.

100. Tsai EC, et al. Reduced plasma peroxyl radical trapping capacity and increased susceptibility of LDL to oxidation in poorly controlled IDDM. Diabetes. 1994;43(8):1010-4.

101. Kawamura M, Heinecke JW, Chait A. Pathophysiological concentrations of glucose promote oxidative modification of low density lipoprotein by a superoxide-dependent pathway. J Clin Investig. 1994;94(2):771.

102. Evans $\mathrm{J}$, et al. Are oxidative stress- activated signaling pathways mediators of insulin resistance and $\beta$-cell dysfunction? Diabetes. 2003;52(1):1-8.

103. Roth $M$. The association of clinical and neurological findings and its bearing on the classification and aetiology of Alzheimer's disease. Br Med Bull. 1986; 42(1):42-50

104. Juhaszova $M$, et al. Protection in the aged heart: preventing the heart-break of old age? Cardiovasc Res. 2005;66(2):233-44.

105. Tani $M$, et al. Direct activation of mitochondrial KATP channels mimics preconditioning but protein kinase $\mathrm{C}$ activation is less effective in middleaged rat hearts. Cardiovasc Res. 2001;49(1):56-68.

106. Jang YC, et al. Increased superoxide in vivo accelerates age-associated muscle atrophy through mitochondrial dysfunction and neuromuscular junction degeneration. FASEB J. 2010;24(5):1376-90.

107. Li F-J, Shen L, Ji H-F. Dietary intakes of vitamin E, vitamin C, and $\beta$-carotene and risk of Alzheimer's disease: a meta-analysis. J Alzheimers Dis. 2012;31(2):253-8.

108. Migliaccio $\mathrm{E}_{\text {, et }}$ al. The p66shc adaptor protein controls oxidative stress response and life span in mammals. Nature. 1999:402(6759):309-13.

109. Giorgio M, et al. Electron transfer between cytochrome $\mathrm{c}$ and p66 Shc generates reactive oxygen species that trigger mitochondrial apoptosis. Cell. 2005;122(2):221-33.

110. Cha M-Y, et al. Mitochondria-specific accumulation of amyloid $\beta$ induces mitochondrial dysfunction leading to apoptotic cell death. PLoS One. 2012; 7(4):e34929.

111. Reddy PH. Amyloid beta, mitochondrial structural and functional dynamics in Alzheimer's disease. Exp Neurol. 2009;218(2):286-92.

112. Canugovi $C$, et al. Base excision DNA repair levels in mitochondrial lysates of Alzheimer's disease. Neurobiol Aging. 2014;35(6):1293-300.

113. Yan MH, Wang $X$, Zhu $X$. Mitochondrial defects and oxidative stress in Alzheimer disease and Parkinson disease. Free Radic Biol Med. 2013;62 90-101.

114. Ahmad W, Ebert PR. Metformin Attenuates Abeta Pathology Mediated Through Levamisole Sensitive Nicotinic Acetylcholine Receptors in a C. elegans Model of Alzheimer's Disease. Mol Neurobiol. 2017;54(7):5427-439.

115. Praticò D, Delanty N. Oxidative injury in diseases of the central nervous system: focus on Alzheimer's disease. Am J Med. 2000;109(7):577-85.

116. Floyd RA. Antioxidants, oxidative stress, and degenerative neurological disorders. Exp Biol Med. 1999;222(3):236-45.

117. Zhu X, et al. Alzheimer disease, the two-hit hypothesis: an update. Biochim Biophys Acta. 2007;1772(4):494-502
118. Wang $X$, et al. Amyloid- $\beta$ overproduction causes abnormal mitochondrial dynamics via differential modulation of mitochondrial fission/fusion proteins. Proc Natl Acad Sci. 2008;105(49):19318-23.

119. Park KW, Baik HH, Jin BK. IL-13-induced oxidative stress via microglial NADPH oxidase contributes to death of hippocampal neurons in vivo. J Immunol. 2009;183(7):4666-74.

120. Baynes JW. Role of oxidative stress in development of complications in diabetes. Diabetes. 1991;40(4):405-12.

121. Hazel JR, Williams EE. The role of alterations in membrane lipid composition in enabling physiological adaptation of organisms to their physical environment. Prog Lipid Res. 1990;29(3):167-227.

122. Prasad MR, et al. Regional membrane phospholipid alterations in Alzheimer's disease. Neurochem Res. 1998;23(1):81-8.

123. Roberts $L$, Morrow JD. Measurement of $F$ 2-isoprostanes as an index of oxidative stress in vivo. Free Radic Biol Med. 2000;28(4):505-13.

124. Stadtman ER. Metal ion-catalyzed oxidation of proteins: biochemical mechanism and biological consequences. Free Radic Biol Med. 1990;9(4):315-25.

125. Smith MA, et al. Widespread peroxynitrite-mediated damage in Alzheimer's disease. J Neurosci. 1997;17(8):2653-7.

126. Good PF, et al. Evidence of neuronal oxidative damage in Alzheimer's disease. Am J Pathol. 1996;149(1):21.

127. Moreira Pl, et al. Alzheimer's Disease and oxidative stress: the old problem remains unsolved. Curr Med Chem Cent Nerv Syst Agents. 2005;5(1):51-62.

128. Aksenov M, et al. Protein oxidation in the brain in Alzheimer's disease. Neuroscience. 2001;103(2):373-83.

129. Bubber $\mathrm{P}$, et al. Mitochondrial abnormalities in Alzheimer brain: mechanistic implications. Ann Neurol. 2005;57(5):695-703.

130. Cao X, et al. Noncoding RNAs in the mammalian central nervous system. Annu Rev Neurosci. 2006;29:77-103.

131. Mehler MF, Mattick JS. Noncoding RNAs and RNA editing in brain development, functional diversification, and neurological disease. Physiol Rev. 2007;87(3):799-823.

132. Nunomura $A$, et al. RNA oxidation in Alzheimer disease and related neurodegenerative disorders. Acta Neuropathol. 2009;118(1):151-66.

133. Bonda DJ, et al. Mitochondrial dynamics in Alzheimer's disease. Drugs Aging. 2010;27(3):181-92.

134. Zhu $X$, et al. Abnormal mitochondrial dynamics in the pathogenesis of Alzheimer's disease. J Alzheimer's Dis. 2013;33(0 1):S253.

135. Zaid $\mathrm{H}$, et al. The voltage-dependent anion channel-1 modulates apoptotic cell death. Cell Death Differ. 2005;12(7):751-60.

136. Ferri KF, Kroemer G. Organelle-specific initiation of cell death pathways. Nat Cell Biol. 2001:3(11):E255-63.

137. Pereira $C$, et al. Cell degeneration induced by amyloid- $\beta$ peptides. J Mol Neurosci. 2004;23(1-2):97-104.

138. Sergeant $N$, et al. Association of ATP synthase a-chain with neurofibrillary degeneration in Alzheimer's disease. Neuroscience. 2003;117(2):293-303.

139. Poon SR, B-K D, Klein JB, Merchant M, Markesbery WR, Butterfield DA. Redox proteomics identification of oxidized proteins in Alzheimer's disease hippocampus and cerebellum: an approach to understand pathological and biochemical alterations in AD. Neurobiol Aging. 2006;27:1564-76.

140. Yan L-J. Positive oxidative stress in aging and aging-related disease tolerance. Redox Biol. 2014;2:165-9.

141. Akter K, et al. Diabetes mellitus and Alzheimer's disease: shared pathology and treatment? Br J Clin Pharmacol. 2011;71(3):365-76.

142. Bomfim TR, et al. An anti-diabetes agent protects the mouse brain from defective insulin signaling caused by Alzheimer's disease-associated $A \beta$ oligomers. J Clin Invest. 2012;122(4):1339-53.

143. Chiti F, Dobson CM. Protein misfolding, functional amyloid, and human disease. Annu Rev Biochem. 2006;75:333-66. 